\title{
Elemental dynamics in hair accurately predict future autism spectrum disorder diagnosis: an international multi-center study
}

\section{Christine Austin}

Icahn School of Medicine https://orcid.org/0000-0002-9966-0492

Paul Curtin

Icahn School of Medicine at Mount Sinai

Manish Arora ( $\square$ manish.arora@mssm.edu)

Icahn School of Medicine at Mount Sinai

Abraham Reichenberg

Icahn School of Medicine at Mount Sinai

Austen Curtin

Mount Sinai School of Medicine

Miyuki Iwai-Shimada3

National Institute for Environmental Studies

Robert Wright

Icahn School of Medicine at Mount Sinai

Rosalind Wright

Icahn School of Medicine at Mount Sinai

Karl Lundin Remnelius

Karolinska Institutet

Johan Isaksson

Karolinska Institutet

Sven Bolte

Karolinska Institutet

Shoji Nakayama

National Institute for Environmental Studies

Article

Keywords:

Posted Date: February 1st, 2022

DOI: https://doi.org/10.21203/rs.3.rs-1307805/v1 
License: (c) (i) This work is licensed under a Creative Commons Attribution 4.0 International License. Read Full License 


\section{Abstract}

Autism spectrum disorder (ASD) is a neurodevelopmental condition diagnosed in approximately $2 \%$ of children. Reliance on the emergence of clinically observable behavioral patterns only delays mean age of diagnosis to approximately 4 years. However, neural pathways critical to language and social functions develop during infancy and current diagnostic protocols miss the age when therapy would be most effective3,4. We developed non-invasive ASD biomarkers using mass spectrometry analysis of elemental metabolism in single hair strands, coupled with machine learning. We undertook a national prospective study in Japan where hair samples were collected at 1 month and clinical diagnosis was undertaken at 4 years. Next, we analyzed a national sample of Swedish twins and, in our third study, participants from a specialist ASD center in the US. In blinded analysis, a predictive algorithm detected ASD risk as early as 1 month with $96.4 \%$ sensitivity, $75.4 \%$ specificity, and $81.4 \%$ accuracy ( $n=486 ; 175$ cases).

\section{Full Text}

Autism spectrum disorder (ASD) is defined by persistent alterations in social communication and interactions alongside restricted, repetitive patterns of behaviors and hyper and hypo sensitivities. ASD is associated with significant impairment in social, occupational, or other important areas of adaptive functioning ${ }^{5}$. Co-occurrence with other neurodevelopmental conditions is common, including attention deficit hyperactivity disorder (ADHD), affecting up to $30 \%$ of children diagnosed with ASD $^{6}$. The US Centers for Disease Control and Prevention (CDC) has reported that the average age of ASD diagnosis in the US is 4 years and 4 months ${ }^{1}$. These results are consistent with global data-a recent meta-analysis (55 cohorts from 35 countries, $n=66,966$ individuals with ASD) found a mean age at diagnosis of 60.48 months (range: $30.90-234.57$ months) ${ }^{7}$. Neural circuits subserving the development of language and related social and sensory pathways including vision and hearing are highly plastic especially during the first year after birth ${ }^{8}$, but the lack of early life diagnostic tools, and the absence of accurate ASD biomarkers that can be applied in infancy, and precede diagnostic assessment based on behavioral phenotypes exclusively, is a major challenge facing ASD therapy development and early intervention delivery ${ }^{9}$. Several randomized trials have reported cognitive and functional gains if therapy is delivered earlier in life ${ }^{3,4,10,11}$. There are also substantial economic benefits of early intervention for ASD with recent estimates suggesting cost savings of $\$ 37$ billion per year in the US alone ${ }^{12}$. However, in the absence of diagnostic biomarkers approved by the European, US and other international regulatory agencies that can detect likelihood of ASD risk in the first year of life, it is not possible to start intervention during the most appropriate critical window of neurodevelopment. Purely genetic approaches have identified a broad range of genetic loci associated with ASD but providing a diagnosis has been challenging because genetic perturbations show marked heterogeneity within ASD cases. Here, we present a hair-based assay that leverages a sequential exposomics platform to detect risk of developing ASD as early as 1 month of age ${ }^{13}$. 
Fetal and infant exposure to toxic metals and deficiencies of nutritional elements have been linked with increased likelihood of ASD and several adverse developmental outcomes frequently associated with ASD, including intellectual disability, and language, attentional and behavioral problems ${ }^{14-16}$. Animal studies show that the effects of various metals on brain development could be mediated through alterations in the regulation of neurotransmission, and altered frontal and subcortical brain structures ${ }^{17}$ several of which have also been implicated in ASD ${ }^{18}$. Further, at the cellular level, studies in animal model systems indicate that essential elements integrated through dietary sources provide critical mediation of receptor functionality in pathways related to autism-like behaviors. ${ }^{19,20}$ Therefore, environmental and dietary exposure to metals and metal metabolism are potentially important etiological factors in $\operatorname{ASD}^{18,21,22}$. Of relevance to the studies we report here, we have previously shown that dysregulation of the dynamics underlying the metabolism of essential and toxic elements is a critical component of ASD etiology 21,22. That work used mass spectrometry analysis of growth increments (growth rings) in teeth to generate a longitudinal profile of elemental metabolism and revealed an alteration in the fine scale time-dependent changes in metal dysregulation (disruption of metal rhythmicity, for example) that increased the risk of ASD. The utility of deciduous teeth as a biomarker of ASD are limited as they are not readily accessible in the first year of life, which is why we have now developed a biomarker utilizing scalp hair. Specifically, for the study we present here, we used a laser ablation-inductively coupled plasma-mass spectrometry analysis of single hair strands to capture the temporal dynamics of metal metabolism. Hair strands grow in an incremental manner at a rate of approximately $1 \mathrm{~cm}$ per month, with some variation by age, gender, race and growth cycle ${ }^{23}$. By rastering a laser along the length of the hair strand and analyzing the ablated material with a mass spectrometer, we generated 4-6 hourly sequential profiles of metal uptake for every participant (Figure 1). Critically, this approach provides indicators of both pharmacodynamic environmental inputs to the developing nervous system, as well as pharmacokinetic processes involved in elemental metabolism.

We undertook studies in three geographically distinct populations to evaluate the accuracy and generalizability of these biomarkers as predictors of ASD ( $n=486,175$ cases). First, we leveraged a prospective population-based nation-wide study in Japan. We collected infant hair at 1 month of age, and DSM-5 clinical diagnosis for ASD was undertaken at 4 years $(n=220$ participants; 110 ASD cases. Details in Methods, Figure 1 and Table 1). Next, to account for underlying heritability, we undertook a populationbased study in Sweden ( $n=138 ; 42$ ASD cases). In this cross-sectional study, twin siblings were evaluated at a single clinical research center for neurodevelopmental conditions. In our third study, we collected hair from patients at a specialist autism center in the US and from a population-based study of neurotypical participants ( $n=128 ; 23$ ASD cases). All studies undertook ASD diagnostic evaluation according to the diagnostic criterial for ASD outlined in the Diagnostic and Statistical Manual for Mental Disorders, 5th edition (DSM-5), of the American Psychiatric Association. To fulfill diagnostic criteria for ASD by using the DSM-5, all three symptoms of social affective difference need to be present in addition to two of four symptoms related to restrictive and repetitive behaviors ${ }^{9}$. We provide additional detail on the presence of intellectual, developmental, psychiatric, and genetic comorbidities in Supplemental Table S3. 
Table 1. Participant characteristics

\begin{tabular}{llllll}
\hline Study & Location & Design & N (cases) & Male/Female & Age, months (SD) \\
JECS & Japan & National prospective study & $220(110)$ & $110 / 110$ & $1(0)$ \\
RATSS & Sweden & National cross-sectional study & $138(42)$ & $76 / 62$ & $170.8(36.9)$ \\
Seaver & USA & Cross-sectional study & $128(23)$ & $80 / 48$ & $61.6(33.4)$ \\
\hline
\end{tabular}

JECS, Japan Environment and Children's Study. RATSS, Roots of Autism and ADHD Twin Study in Sweden. Seaver, Seaver Autism Center Study. Reported age indicates participant age at sample collection.

Laboratory analysis of a single hair strand yields timeseries measurements for 15 elements. For each individual element, we used recurrence quantification analysis (RQA) to measure the variability in discrete elemental signals over time; and applied a complementary method, cross-recurrence quantification analyses (CRQA), to measure cross-elemental temporal dynamics (see Methods for details). We have previously used this computational approach to timeseries data of elemental uptake to quantify characteristics of metabolic dynamics, including rhythmicity, complexity, and stability ${ }^{24}$. Overall, this process yields 210 discrete quantitative features for each elemental pathway, accounting for the dynamics underlying the metabolism of single elements as well as pairwise dynamics across multiple elements.

The laboratory analysis of hair requires stringent quality control and quality assurance (QA/QC) protocols. We also confirmed the reproducibility of hair element measurements by analyzing two hair samples collected from the same participant at one time point and further confirmed the stability of our elemental measures by analyzing the RQA features in hair samples collected 5 years apart in a subsample. Our results indicated a high level of reproducibility (within $+/-10 \%$ ) for replicate measures at the same timepoint as well as at replicate measures taken years apart (see Supplemental Material).

Our initial computational analysis focused on discrete associations between individual biomarkers and ASD diagnosis. In Figure 2A we show the results of this analysis, wherein we identified 567 individual features which significantly differed between ASD cases and controls after adjustment for age and sex, and false discovery rate (FDR) correction for multiple comparisons. Two general patterns emerged from this analysis. First, our findings indicate a broad pattern of dysregulated elemental dynamics which spanned every elemental pathway investigated. This was apparent from the representation of all 15 elements and the even spread of features with positive and negative effects; a pattern generally indicative of bidirectional effects suggestive of broad dysregulation. Second, in a subset of essential and nonessential elements, including zinc, lithium, and copper, these effects were almost entirely unidirectional, with ASD cases exhibiting attenuated periodic dynamics relative to controls. These findings are in agreement with earlier epidemiologic reports that have shown a systemic changes to the regulation of elements in $\mathrm{ASD}^{21,25,26}$, particularly zinc and copper, and have been supported by mechanistic findings in animal models 27,28 . 
Next, we sought to leverage these patterns in the construction of an ensemble model to predict ASD case status. Data were combined from all populations and randomly portioned in a training set ( $80 \%$ of data; $\mathrm{N}=389,147$ ASD cases) for model training, and a validation set ( $\mathrm{N}=97 ; 28$ ASD cases) to evaluate model performance. Figure 2B shows the receiver operator characteristic $(\mathrm{ROC})$ curve derived from predicting ASD case status in the validation set. At optimal criterion, this model yielded $96 \%$ (95\% Cl: $82-100 \%)$ sensitivity, 75\% (95\%Cl: 64-85\%) specificity, and 81\% (95\% Cl: 72-89\%) overall accuracy. A full confusion matrix is provided in Supplemental Table S2.

In clinical applications, predictive efficacy is typically assessed through the estimation of positive predictive value (PPV) and negative predictive value (NPV). Relative to the prevalence of ASD in our validation dataset (28\%) due to use of cohorts with enriched risk, the positive predictive value (PPV) of this device was $60.3 \%$ (50.0-69.8\%), while the negative predictive value (NPV) was $98.1 \%$ (88.7-99.7\%). We also considered these estimates after adjustment for the population-based prevalence via Bayes Theorem, with the target population in this case assumed to be enriched-risk children where prevalence is estimated at $17 \%$ (see discussion below for this estimate). ${ }^{29}$ Relative to this prevalence, device PPV is $44.5 \%$ (34.5-54.9\%) and NPV is $99.0 \%$ (93.7-99.9\%). In practice, the robust NPV of this device would ensure that negative results could be interpreted with a high level of confidence despite the sparse prevalence of the disease.

We also considered how device performance varied across key demographic and biological factors including age and sex. In Figure 2C, we provide sex-stratified indicators of device performance for participants in the holdout validation dataset. In general, sex-stratified model performance, summarized as AUC, did not differ from overall model performance for male $(P=0.98)$ and female $(P=0.88)$ participants. Likewise, as shown in Figure 2D, when stratifying participants in age categories relative to early life, pre-adolescence, and adolescence, we found that age-specific estimates of device performance did not significantly differ from overall device performance.

It is desirable to identify objective biomarkers that can predict the emergence of ASD before the development of behavioral symptoms and support early intervention. Current efforts to achieve these goals have focused on genomic loci, but multiple lines of evidence consistently implicate environmental associations with the etiology of ASD. ${ }^{18,30}$ Here, we developed a novel analytical paradigm that leverages high-resolution longitudinal sampling to measure temporal dynamics in elemental uptake and metabolism, thereby leveraging signatures of both the internal and external environment. This approach allowed the generation of a model which accurately predicts the later emergence of ASD from samples collected from children as young as 1 month of age.

The development of this paradigm builds from several recent studies which employed similar approaches. In previous work, we identified signatures in elemental metabolism that were highly predictive of ASD status 22 ; however, though that study's analytical methods were largely similar to those employed here, it did not include prospectively collected samples, and the tissue matrix investigated (teeth) is not accessible soon after birth as is hair. Likewise, a related study showed that temporal 
dynamics in elemental metabolism were effective in discriminating samples collected from neurotypical children, and those with ASD, ADHD, or comorbid ASD/ADHD diagnoses ${ }^{21}$. In extending these methods to the analysis of hair samples, and applying this approach to the analysis of prospectively collected samples, the present study thus builds on prior established work to generate a novel hair-based ASD biomarker.

Both the strengths and limitations must be considered when interpreting the results of this study. The ethnically and geographically diverse study populations assessed here support broad biological generalizability, but future studies are needed to replicate and refine the predictive algorithms generated here to develop an effective medical diagnostic device. The prevalence of ASD in our study population was $28 \%$. This is substantially higher than the 2 to $3 \%$ prevalence in the general population of children but closer to the prevalence observed in groups with high likelihood of ASD. For example, based on the estimates of Hansen and colleagues who studied 2.5 million births in six countries for the risk of ASD when an older sibling has an ASD diagnosis, the prevalence estimates would be between $9 \%$ and $17 \%$ when a full sibling has $\mathrm{ASD}^{29}$, and even higher when multiple risk factors co-exist (for example, $29 \%$ of children with $A D H D$ also have $A S D^{31}$ ). Advancing paternal and maternal age have both been associated with ASD. Specifically, for fathers 45 years or older the relative risk of having a child with ASD is $30-50 \%$ higher than fathers who are 20-29 years of age ${ }^{32}$. Increasing maternal age and a mismatch in maternal and paternal age are also associated with increased risk of having a child with ASD $^{32,33}$. Infants and children with ASD are known to fall behind on developmental milestones ${ }^{34}$. Several clinical tools are available such as Modified-CHecklist for Autism in Toddler (M-CHAT), First Years Inventory (FYI), and Quantitative-CHecklist for Autism in Toddler (Q-CHAT), which may be used by clinicians to evaluate children. However, they have low-to-moderate accuracy when used alone and would benefit from additional information provided by a biomarker-based diagnostic aid ${ }^{35}$. Overall, the biomarker we propose here must be viewed as a diagnostic aid that can assist in early detection of ASD in conjunction with a thorough clinical evaluation.

The sensitivity and specificity estimates we report here must be considered in relation to the background prevalence of ASD in the enriched-risk populations where the hair biomarker could appropriately be used. The NPV of the device in this diverse sample population suggests that this approach can effectively provide negative diagnostic indicators in diverse clinical conditions with varying disease prevalence; however, positive diagnostic indicators will need to be cautiously interpreted, and will decline in utility as the device is applied to broader populations where prevalence is reduced. One potential solution to this challenge would be the extension of the single-sample method applied here to the evaluation of multiple samples. By analyzing three hairs in tandem, for example, and requiring 3 consistent "positive" results to serve as criterion for positive diagnosis, PPV could be increased to $94 \%$, assuming other factors remain constant. Future studies are needed to evaluate the potential benefits of such an ensemble; and, more generally, to validate and confirm that these features provide stable diagnostic performance across larger and more diverse populations. Likewise, the stability of the features measured here should be assessed through repeat prospective sampling over the course of the lifespan, and future studies should evaluate 
the efficacy of this approach in predicting ASD in adults. We have, however, shown that the elemental dynamics in hair that are relevant to ASD diagnosis are highly stable using repeat samples collected from the same participants 5 years apart (see Supplemental Material). Of note, our analysis of hair focuses on

the dynamics of elemental metabolism as measured in hair and is not reliant on concentrations of metals in hair. The integration of a prospective study design, wherein samples were collected soon after birth, prior to the development of symptoms and later diagnosis, is a particular strength of this study which future studies should emulate.

Overall, we report a novel integrated platform which combines high-resolution longitudinal sampling of essential, non-essential, and toxic elements in hair with a machine learning ensemble for the prediction of ASD case status. Applying computational methods derived from chaos theory, particularly RQA and CRQA, we characterized single and across-element temporal dynamics that underlie ASD. Our approach contrasts previous efforts that relied solely on genetic readouts to identify ASD biomarkers. Instead, we have focused on the interface of genetically driven metabolic signatures with external environmental exposures thus considering both the genetic origins and the environmental triggers that lead to ASD. The biomarker we propose here holds the promise to improve the lives of ASD-affected individuals with diagnosis at a younger age that facilitates early intervention.

\section{Online Methods}

\section{Study populations}

Our participants were recruited from four different studies being undertaken in three countries. Ethics clearances were obtained from the relevant institutional review boards at the coordinating site of each study. Key characteristics of the studies are outlined in Table 1.

The Japan Environment and Children's Study. This national Japanese study also known as JECS, is a nationwide birth cohort study investigating environmental factors that might affect children's health and development ${ }^{36,37}$. Fifteen Regional Centers located throughout Japan were responsible for recruiting women in early pregnancy living in their respective recruitment areas. Self-administered questionnaires and medical records were used to obtain such information as demographic factors, lifestyle, socioeconomic status, environmental exposure, medical history, and delivery information. In the period up to delivery, we collected bio-specimens, including hair.

The total number of pregnancies resulting in delivery was 100,778 , of which $51,402(51.0 \%)$ involved program participation by male partners. Discounting pregnancies by the same woman, the study included 95,248 unique mothers and 49,189 unique fathers. The 100,778 pregnancies involved a total of 101,779 fetuses and resulted in 100,148 live births. The coverage of children in 2013 (the number of live births registered in JECS divided by the number of all live births within the study areas) was approximately $45 \%$. Nevertheless, the data on the characteristics of the mothers and children we studied showed marked similarity to those obtained from Japan's 2013 Vital Statistics Survey. 
We selected 220 participants randomly from the 82,413 who responded to the 3-year-old questionnaire (JECS dataset, jecs-ta-20190930). The random selection was based on the following criteria:

1) Hair sample collected at 1 month of age and 1 strand available for analysis

2) Case selection: randomly selected from those with confirmed autism spectrum disorder diagnosis $(n=372)$

3) Control selection: randomly selected to match cases on age (within same year), gender and province (location) $(\mathrm{n}=82,041)$.

Hair was collected from children at the age of 1 month, stored in ziplock storage plastic bags with alphanumeric barcodes and housed at the JECS biospecimen repository after being labeled. JECS participants are evaluated at 6-monthly to half-yearly intervals. At age 4, neurodevelopmental assessments are recorded including a confirmed ASD diagnosis from a health care provider. Subsequently, to confirm the accuracy of the diagnosis, the medical record for each participant abstracted by a board-certified pediatric psychiatrist and a DSM- 5 criteria diagnosis of ASD is confirmed.

Roots of Autism and ADHD Twin Study (RATSS), Sweden. Participating twins in this study are part of RATSS recruited between 2011 and $2016^{38}$. The study was approved by the Swedish Regional Ethical Review Board and all participants gave written informed consent. Potential twin participants for the RATSS are identified through nationwide registries, including the Child and Adolescent Twin Study in Sweden (CATSS) ${ }^{39}$, a population-based study of all twins born in Sweden since 1992 in which all twins are screened at age nine using the Autism, Tics, ADHD and other disorders using Comorbidities Inventory (A-TAC). Participants are identified through linking the Swedish Twin Registry to other National registries such as the Swedish National Patient Register, and regional clinical registers in Stockholm County (Child and Adolescent Psychiatry ["Pastill"], Habilitation \& Health Centers) that include ICD-10 diagnostic information ${ }^{40,41}$. Finally, potential participants are also identified through Swedish societies for neurodevelopmental disorders as well as advertisements and summons in the media. Even though the recruitment is done through different routes, $>80 \%$ of the twins in RATSS are present in the Swedish twin registries.

Twin pairs were recruited into the RATSS either based on discordance for ASD ( $>2$ points differences on the A-TAC autism subscale equaling 1 SD); concordance for ASD (both twins reaching cut-off on the ATAC autism scale); or concordance for no NDD (both twins under cut-offs for all NDD subscale on the ATAC). For other sources of recruitment, the twins are invited if at least one twin has an ICD-10 diagnosis of autism (F84.0), Asperger syndrome (F84.5), or atypical autism/pervasive developmental disorder not otherwise specified (PDD-NOS) (F84.1, F84.8, F84.9), or a Diagnostic and Statistical Manual of Mental Disorders Fifth Edition (DSM-5) diagnosis of ASD (either parent- or registry reported). All potential participants undergo a telephone interview by a research nurse checking eligibility before the invitation for assessment in the RATSS. ASD was diagnosed according to DSM-5 criteria during a $2 \frac{1}{2}$ day visit at a clinical research unit based on clinical experts consensus and corroborated by results from first choice 
standardized diagnostic tools such as Autism Diagnostic Interview - Revised (ADI-R) and the Autism Diagnostic Observation Schedule Second Edition (ADOS-2). Zygosity was determined by genotyping of saliva, or whole-blood derived DNA using standard methods. The genotyping was done using Infinium Human-CoreExome chip (Illumina Inc. USA). The estimating identity by descent was analyzed using the PLINK software (v1.07) ${ }^{42}$ after quality control and removal of SNPs with minor allele frequency less than 0.05 within the samples. All pairs of DNA samples showing $>=0.99$ were considered as monozygotic pairs. Medical history and sociodemographic information of the families were collected.

Seaver Autism Center, and PRISM Study, Mount Sinai Hospital, New York, USA. The Seaver Autism Center is located at the Icahn School of Medicine at Mount Sinai in New York City and serves a diverse and complex patient population. The Seaver Center has longstanding community ties and receives approximately 600 new autism referrals annually for research and/or clinical services. Ethical approval for the study was obtained from Mount Sinai School of Medicine research ethics committee. All participants and/or their parents provided written informed consent. In 2016 we contacted all families in ongoing studies and services at the Seaver Center that ${ }^{43}$ had a child with ASD as well as an additional child without a diagnosis of ASD. Informed consent was obtained from all parents or legal guardians. ASD diagnosis was confirmed through a gold-standard evaluation including the ADOS-2, and the ADI-R and a clinical evaluation with a board-certified child and adolescent psychiatrist or licensed clinical psychologist to assess DSM-5 criteria for ASD. We supplemented this sample with population-based controls from the PRogramming of Intergenerational Stress Mechanisms (PRISM) study that enrolls children of mothers receiving prenatal care from Mount Sinai Hospital.

\section{Laboratory Methods}

Single hair strands from each participant were washed to remove surface contaminants in a solution of $1 \%$ Triton X-100 and ultra-pure water $\left(18.2 \mathrm{M} \Omega \mathrm{cm}^{-1}\right)$ by sonication for 1 minute. Hairs were then rinsed with ultra-pure water to remove the surfactant and dried in an oven at $60 \otimes \mathrm{C}$ overnight. Hairs were mounted on plain glass microscope slides using double sided tape and loaded into the ablation cell. A New Wave Research NWR-193 (ESI, USA) laser ablation unit equipped with a 193nm ArF excimer laser was connected to an Agilent Technologies 8800 triple-quad ICP-MS (Agilent Technologies, USA) for elemental analysis. Helium was used as a carrier gas from the laser ablation cell and mixed with argon via Y-piece before introduction to the ICP-MS. The system was tuned daily using NIST SRM 612 (trace elements in glass) to monitor sensitivity (maximum analyte ion counts), oxide formation $\left({ }^{232} \mathrm{Th}^{16} \mathrm{O}^{+} /{ }^{232} \mathrm{Th}^{+},<0.3 \%\right)$ and fractionation $\left({ }^{232} \mathrm{Th}^{+} /{ }^{238} \mathrm{U}^{+}, 100 \pm 5 \%\right)$. A pre-ablation scan at low laser energy (0.27-0.32 $\left.\mathrm{Jcm}^{-2}\right)$ was first run along the hair to remove the surface layer and reduce contamination of the endogenous signal. The hair was scanned again along the same path at higher energy $\left(0.50-0.55 \mathrm{Jcm}^{-2}\right)$ to collect element signal intensity along the strand. A path of approximately $10 \mathrm{~mm}$ was scanned along each hair, representing about 1 month of growth, and providing over 650 sampling points. Data were analyzed as element to sulfur ratios (e.g. $\left.{ }^{66} \mathrm{Zn}:{ }^{34} \mathrm{~S}\right)$ to control for any variations in the density within a hair and between samples. 


\section{Computational Methods}

The computational analysis of elemental time series involved three successive phases of feature engineering, statistical analysis, and predictive modeling. The feature engineering stage involved the application of signal processing methods, particularly Recurrence Quantification Analysis (RQA) and cross-recurrence quantification analysis (CRQA), to derive descriptive statistics (features) for each element, and to measure relationships between elements. The second phase of computational analysis involved a features-wide association analysis, conceptually similar to a genome-wide association study (GWAS), which tested for associations between measured features and ASD diagnostic status. The final stage of analysis involved the training of a machine learning ensemble to leverage descriptive features to predict ASD case status.

\section{Feature Engineering}

In the feature engineering stage of processing, for each of 15 elemental time series measured in each hair, and for the pairwise interactions between each elemental time series, a recurrence matrix or crossrecurrence matrix, respectively, was generated to reconstruct underlying signal dynamics ${ }^{44,45}$. Following the approach developed in prior studies ${ }^{21,22,24,46}$ utilizing elemental time series, the delay $(\tau)$ and embedding dimension $(m)$ parameters involved in recurrence plot construction were determined through the minimization of mutual information and false-nearest neighbor algorithms, respectively; likewise, to facilitate cross-subject comparison, threshold functions, $\epsilon$, were constrained to yield recurrence rates to $10 \%$. From each recurrence or cross-recurrence matrix thereby derived from each sample, an array of quantitative metrics was calculated via RQA/CRQA; the estimation and interpretation of these features are summarized in Supplemental Table S1. The general rationale for this framework is to derive descriptive statistics which characterize temporal dynamics in elemental time series.

More specifically, the rationale for the use RQA/CRQA to analyze elemental time series derives from prior studies utilizing similar approaches, as these methods offer a number of advantages in this application. First, the derivation of descriptive statistics ("features") based on signal dynamics, rather than momentary signal intensity, provides a means of measuring within-signal dynamics, which previous studies have shown are consistent across populations even when populations have varying levels of concentration ${ }^{47}$. Second, unlike related signal decomposition techniques, such as Fourier analysis or Wavelet transformations, the application of RQA is robust in the presence of noise, applicable in comparatively short time series (relative to Fourier/Wavelet), and robust against non-stationarity in the data $^{48}$. Third, prior studies which have applied RQA/CRQA to the longitudinal analysis of essential and non-essential elements, as are utilized in this device, have shown, as noted, that RQA yields robustly generalizable measures of elemental metabolism; and, further, that these parameters are highly sensitive

to systemic disease states, including autism spectrum disorder ${ }^{49}$, attention deficit hyperactivity disorder $^{21}$, and amyotrophic lateral sclerosis ${ }^{46}$. Relevant to ASD, specifically, two prior studies have utilized RQA to identify ASD-related dysregulation of elemental metabolism; and have utilized RQA-based 
features in the analysis of longitudinal elemental exposures to generate predictive classifiers for ASD which were highly accurate ${ }^{21,49}$.

In sum, the application of RQA to individual elemental timeseries, and the application of CRQA to characterize pair-wise dynamics between each potential elemental pairings, yields for each time-series (and pairing of time-series) 12 descriptive features which characterize signal dynamics; in particular, the prevalence, duration, timing, and complexity of stable and periodic states.

\section{Statistical Analysis}

The inferential analysis of RQA/CRQA features derived from each element timeseries, and from pairwise dependencies among elements, involved the construction of discrete generalized linear models to test for associations between RQA/CRQA features and ASD diagnostic status. For each feature, a discrete linear model was constructed to test for differences in RQA/CRQA features between ASD cases and controls. Models were adjusted for child sex and age at sample collection. P-values associated with elemental features were corrected for multiple comparisons with false discovery rate (FDR) adjustment. Features were batch-corrected and normalized ( $\mathrm{z}$-scored) prior to statistical analysis ${ }^{50}$; to meet criterion of statistical significance, FDR values were required to be less than 0.05 .

\section{Predictive Modeling}

The goal of the predictive modeling was to utilize descriptive statistics ("features") generated in the descriptive analysis of elemental time series to predict ASD case status. The model utilized for predictive classification was a form of ensemble gradient boosting introduced by Chen and Guestrin ${ }^{51}$, referred to as XGBoost ("Extreme Gradient Boosting"). For model training, $80 \%$ of the data ( $\mathrm{N}=389 ; 147$ ASD cases) were selected with random assignment. A proprietary subset of features were utilized in model training. Models were then tuned with 5-fold cross validation in the training set, with 1000 iterations of modelbased optimization, per the procedure developed by Bichi et al. ${ }^{46}$, to identify the best-performing set of hyperparameters.. Following hyperparameter tuning, the selected set of hyperparameters was used to fit a model across the full set of training data. The performance of this model was then evaluated by predicting case status in the remaining holdout dataset, comprising $20 \%$ of the total data ( $N=97 ; 28$ ASD), and comparing the alignment of predicted case status with case status as it was assigned by the current gold standard reference of formal clinical diagnosis of ASD according to DSM-5.

\section{Software Implementation}

Feature engineering, including implementation of RQA/CRQA, was done in the Julia (v1.5.2) programming language utilizing the DynamicalSystems.jl library ${ }^{52}$. Visualization of recurrence matrices and some related functions were done with the Cross-Recurrence Toolbox in Matlab. Statistical analyses and predictive modeling were implemented in the R (v. 3.6.3) programming language. The "data.table" (v1.13.2) library was used for data manipulation; the "broom" (v0.7.1) library was used for statistical analyses and feature selection; the "pROC" (v1.16.2) and "ggplot2" (v3.3.2) libraries were used in 
visualizing model performance via receiver operating characteristic (ROC) curves; and, the " $m \mid r$ " (v2.19.22), "xgboost" (v1.2.0.1), and "caret" (v6.0-86) packages were used in model training, fitting, and prediction.

\section{Declarations}

\section{Conflict of interest:}

Manish Arora, Christine Austin and Paul Curtin are affiliated with Linus Biotechnology Inc., a start-up company of the Mount Sinai Health System. They hold equity in the company. The company develops tools for the detection of autism spectrum disorder and related conditions.

\section{References}

1. Maenner MJ, Shaw KA, Baio J, et al. Prevalence of Autism Spectrum Disorder Among Children Aged 8 Years - Autism and Developmental Disabilities Monitoring Network, 11 Sites, United States, 2016. MMWR Surveill Summ. 2020;69(4):1-12.

2. $\quad$ Autism Spectrum Disorders in the European Union (ASDEU). 2018.

3. Dawson G, Rogers S, Munson J, et al. Randomized, controlled trial of an intervention for toddlers with autism: the Early Start Denver Model. Pediatrics. 2010;125(1):e17-23.

4. Green J, Charman T, McConachie $\mathrm{H}$, et al. Parent-mediated communication-focused treatment in children with autism (PACT): a randomised controlled trial. Lancet. 2010;375(9732):2152-2160.

5. de Schipper E, Lundequist A, Coghill D, et al. Ability and Disability in Autism Spectrum Disorder: A Systematic Literature Review Employing the International Classification of Functioning, Disability and Health-Children and Youth Version. Autism Res. 2015;8(6):782-794.

6. Simonoff E, Pickles A, Charman T, Chandler S, Loucas T, Baird G. Psychiatric disorders in children with autism spectrum disorders: prevalence, comorbidity, and associated factors in a population-derived sample. J Am Acad Child Adolesc Psychiatry. 2008;47(8):921-929.

7. van 't Hof $\mathrm{M}$, Tisseur $\mathrm{C}$, van Berckelear-Onnes I, et al. Age at autism spectrum disorder diagnosis: A systematic review and meta-analysis from 2012 to 2019 . Autism. 2021;25(4):862-873.

8. Shonkoff JP, Phillips DA. From Neurons to Neighborhoods: The Science of Early Childhood Development. In: Development NRCUaloMUColtSoEC, ed. Washington (DC): National Academies Press (US); 2000.

9. Hyman SL, Levy SE, Myers SM, Council On Children With Disabilities SOD, Behavioral P. Identification, Evaluation, and Management of Children With Autism Spectrum Disorder. 
Pediatrics. 2020;145(1).

10. Pickles A, Le Couteur A, Leadbitter K, et al. Parent-mediated social communication therapy for young children with autism (PACT): long-term follow-up of a randomised controlled trial. Lancet. 2016;388(10059):2501-2509.

11. Sinai-Gavrilov Y, Gev T, Mor-Snir I, Vivanti G, Golan O. Integrating the Early Start Denver Model into Israeli community autism spectrum disorder preschools: Effectiveness and treatment response predictors. Autism. 2020;24(8):2081-2093.

12. Frazier TW, Coury DL, Sohl K, et al. Evidence-based use of scalable biomarkers to increase diagnostic efficiency and decrease the lifetime costs of autism. Autism Res. 2021;14(6):1271-1283.

13. Branca M. Slivers of the spectrum. Nat Biotechnol. 2021;39(5):540-545.

14. Sanders AP, Claus Henn B, Wright RO. Perinatal and Childhood Exposure to Cadmium, Manganese, and Metal Mixtures and Effects on Cognition and Behavior: A Review of Recent Literature. Current Environmental Health Reports. 2015;2(3):284-294.

15. von Stackelberg K, Guzy E, Chu T, Claus Henn B. Exposure to Mixtures of Metals and Neurodevelopmental Outcomes: A Multidisciplinary Review Using an Adverse Outcome Pathway Framework. Risk Anal. 2015;35(6):971-1016.

16. Grandjean P, Landrigan PJ. Developmental neurotoxicity of industrial chemicals. The Lancet. 2006;368(9553):2167-2178.

17. Modabbernia A, Arora M, Reichenberg A. Environmental exposure to metals, neurodevelopment, and psychosis. Current Opinion in Pediatrics. 2016;28(2):243-249.

18. Grabrucker AM. Environmental factors in autism. Front Psychiatry. 2012;3:118.

19. Ha HTT, Leal-Ortiz S, Lalwani K, et al. Shank and Zinc Mediate an AMPA Receptor Subunit Switch in Developing Neurons. Front Mol Neurosci. 2018;11:405.

20. Shih PY, Hsieh BY, Lin MH, et al. CTTNBP2 Controls Synaptic Expression of Zinc-Related AutismAssociated Proteins and Regulates Synapse Formation and Autism-like Behaviors. Cell Rep. 2020;31(9):107700.

21. Austin C, Curtin P, Curtin A, et al. Dynamical properties of elemental metabolism distinguish attention deficit hyperactivity disorder from autism spectrum disorder. Translational psychiatry. 2019;9(1):238.

22. Curtin P, Austin C, Curtin A, et al. Dynamical features in fetal and postnatal zinc-copper metabolic cycles predict the emergence of autism spectrum disorder. Sci Adv. 2018;4(5):eaat1293. 
23. Robbins CR. Chemical and Physical Behavior of Human Hair. fifth ed: Springer; 2012.

24. Curtin P, Curtin A, Austin C, et al. Recurrence quantification analysis to characterize cyclical components of environmental elemental exposures during fetal and postnatal development. PLOS One. 2017;12(11):e0187049.

25. Arora $\mathrm{M}$, Reichenberg $\mathrm{A}$, Willfors $\mathrm{C}$, et al. Fetal and postnatal metal dysregulation in autism. Nat Commun. 2017;8:15493.

26. Curtin P, Austin C, Curtin A, et al. Dynamical features in fetal and postnatal zinc-copper metabolic cycles predict the emergence of autism spectrum disorder. Sci Adv. 2018;4(5):eaat1293.

27. Grabrucker AM. A role for synaptic zinc in ProSAP/Shank PSD scaffold malformation in autism spectrum disorders. Dev Neurobiol. 2014;74(2):136-146.

28. Grabrucker S, Jannetti L, Eckert M, et al. Zinc deficiency dysregulates the synaptic ProSAP/Shank scaffold and might contribute to autism spectrum disorders. Brain. 2014;137(Pt 1):137-152.

29. Hansen SN, Schendel DE, Francis RW, et al. Recurrence Risk of Autism in Siblings and Cousins: A Multinational, Population-Based Study. J Am Acad Child Adolesc Psychiatry. 2019;58(9):866-875.

30. Pugsley K, Scherer SW, Bellgrove MA, Hawi Z. Environmental exposures associated with elevated risk for autism spectrum disorder may augment the burden of deleterious de novo mutations among probands. Mol Psychiatry. 2021.

31. Rao PA, Landa RJ. Association between severity of behavioral phenotype and comorbid attention deficit hyperactivity disorder symptoms in children with autism spectrum disorders. Autism. 2014;18(3):272-280.

32. Sandin S, Schendel D, Magnusson P, et al. Autism risk associated with parental age and with increasing difference in age between the parents. Mol Psychiatry. 2016;21(5):693-700.

33. Sandin S, Hultman CM, Kolevzon A, Gross R, MacCabe JH, Reichenberg A. Advancing maternal age is associated with increasing risk for autism: a review and meta-analysis. J Am Acad Child Adolesc Psychiatry. 2012;51(5):477-486.e471.

34. Matson JL, Mahan S, Kozlowski AM, Shoemaker M. Developmental milestones in toddlers with autistic disorder, pervasive developmental disorder-not otherwise specified and atypical development. Dev Neurorehabil. 2010;13(4):239-247.

35. Petrocchi S, Levante A, Lecciso F. Systematic Review of Level 1 and Level 2 Screening Tools for Autism Spectrum Disorders in Toddlers. Brain Sci. 2020;10(3). 
36. Michikawa T, Nitta H, Nakayama SF, et al. Baseline Profile of Participants in the Japan Environment and Children's Study (JECS). J Epidemiol. 2018;28(2):99-104.

37. Kawamoto T, Nitta H, Murata $\mathrm{K}$, et al. Rationale and study design of the Japan environment and children's study (JECS). BMC Public Health. 2014;14:25.

38. Bolte S, Willfors C, Berggren S, et al. The Roots of Autism and ADHD Twin Study in Sweden (RATSS). Twin Res Hum Genet. 2014;17(3):164-176.

39. Anckarsater $\mathrm{H}$, Lundstrom S, Kollberg L, et al. The Child and Adolescent Twin Study in Sweden (CATSS). Twin Res Hum Genet. 2011;14(6):495-508.

40. Lichtenstein P, De Faire U, Floderus B, Svartengren M, Svedberg P, Pedersen NL. The Swedish Twin Registry: a unique resource for clinical, epidemiological and genetic studies. $J$ Intern Med. 2002;252(3):184-205.

41. Pedersen NL, Lichtenstein P, Svedberg P. The Swedish Twin Registry in the third millennium. Twin Res. 2002;5(5):427-432.

42. Purcell S, Neale B, Todd-Brown K, et al. PLINK: a tool set for whole-genome association and population-based linkage analyses. Am J Hum Genet. 2007;81(3):559-575.

43. Siper PM, Kolevzon A, Wang AT, Buxbaum JD, Tavassoli T. A clinician-administered observation and corresponding caregiver interview capturing DSM-5 sensory reactivity symptoms in children with ASD. Autism Research. 2017;10(6):1133-1140.

44. Webber CL, Zbilut JP. Dynamical Assessment of Physiological Systems and States Using Recurrence Plot Strategies. J Appl Physiol. 1994;76(2):965-973.

45. Marwan N, Romano MC, Thiel M, Kurths J. Recurrence plots for the analysis of complex systems. Phys Rep. 2007;438(5-6):237-329.

46. Curtin $P$, Austin $C$, Curtin $A$, et al. Dysregulated biodynamics in metabolic attractor systems precede the emergence of amyotrophic lateral sclerosis. PLoS Comput Biol. 2020;16(4):e1007773.

47. Curtin $\mathrm{P}$, Curtin $\mathrm{A}$, Austin $\mathrm{C}$, et al. Recurrence quantification analysis to characterize cyclical components of environmental elemental exposures during fetal and postnatal development. PLoS One. 2017;12(11):e0187049.

48. Zbilut JP, Giuliani A, Webber CL. Recurrence quantification analysis and principal components in the detection of short complex signals. Phys Lett A. 1998;237(3):131-135.

49. Curtin P, Austin C, Curtin A, et al. Dynamical features in fetal and postnatal zinc-copper metabolic cycles predict the emergence of autism spectrum disorder. Science Advances. 2018;4(5):eaat1293. 
50. Zhang Y, Parmigiani G, Johnson WE. ComBat-seq: batch effect adjustment for RNA-seq count data. NAR Genom Bioinform. 2020;2(3):Iqaa078.

51. Chen T, Guestrin C. XGBoost: A Scalable Tree Boosting System. Paper presented at: KDD '16: Proceedings of the 22nd ACM SIGKDD International Conference on Knowledge Discovery and Data Mining; August, 2016; San Francisco, USA.

52. Datseris G. DynamicalSystems.jl: A Julia software library for chaos and nonlinear dynamics. Journal of Open Source Software. 2018;3(23):598.

\section{Figures}



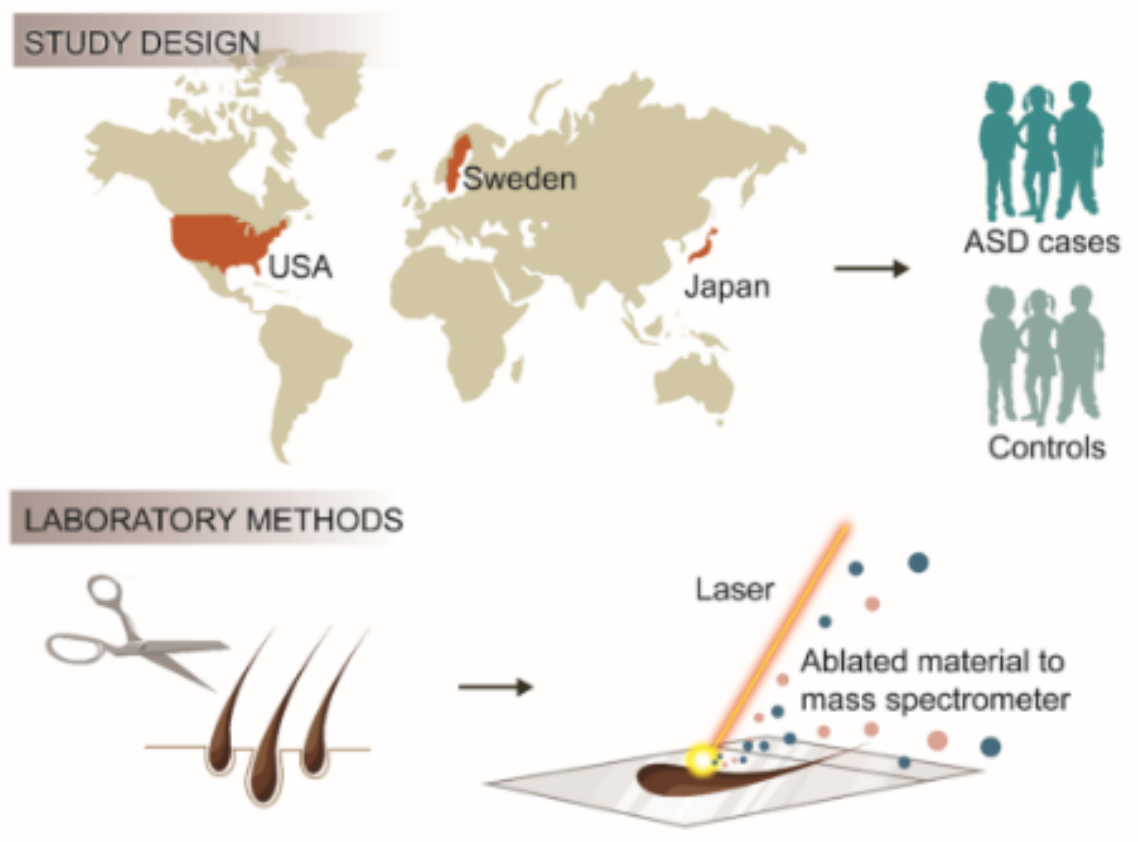

\section{DATA SCIENCE}

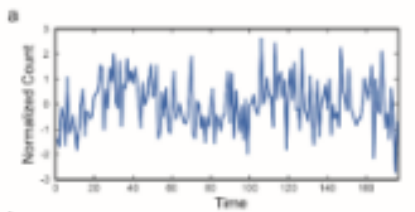

b
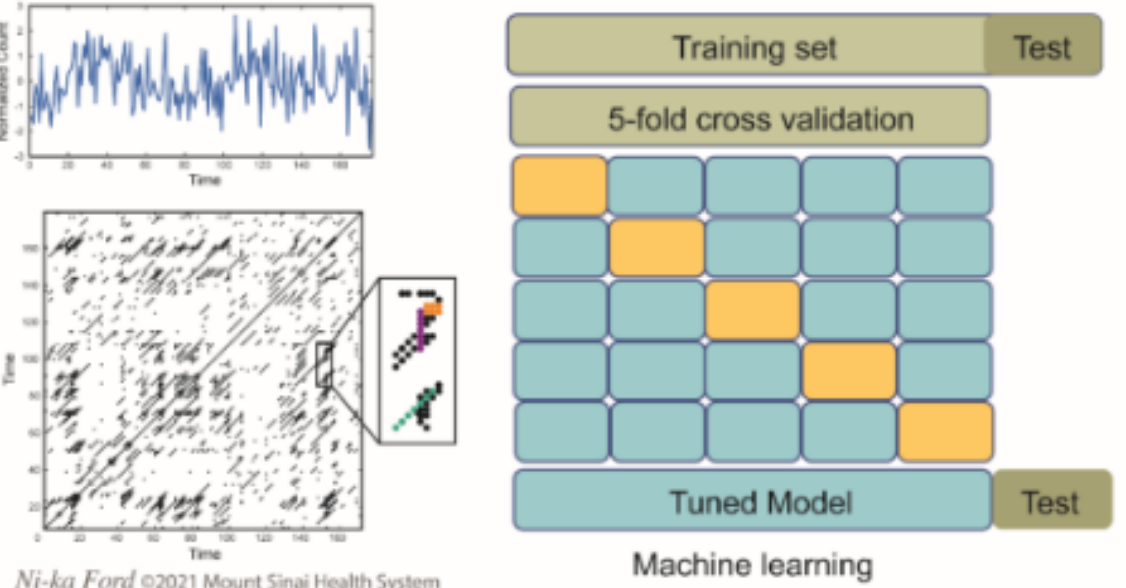

Figure 1. Study Design and Analytical Pipeline. Participants were recruited in Japan (prospective national study), Sweden (cross-sectional national study) and New York (cross-sectional single clinical center study). Clinical case ascertainment was undertaken using DSM-5 criteria. Scalp hair strands were analyzed using laser ablation-inductively coupled plasma-mass spectrometry to generate 4 to 6 hourly profiles of elemental uptake. Recurrence and cross-recurrence quantification analysis was used to quantify the dynamic nature of elemental assimilation. A machine learning algorithm was trained on $80 \%$ of the data and tested on a randomly selected $20 \%$ hold-out set.

\section{Figure 1}

See image above for figure legend. 
A

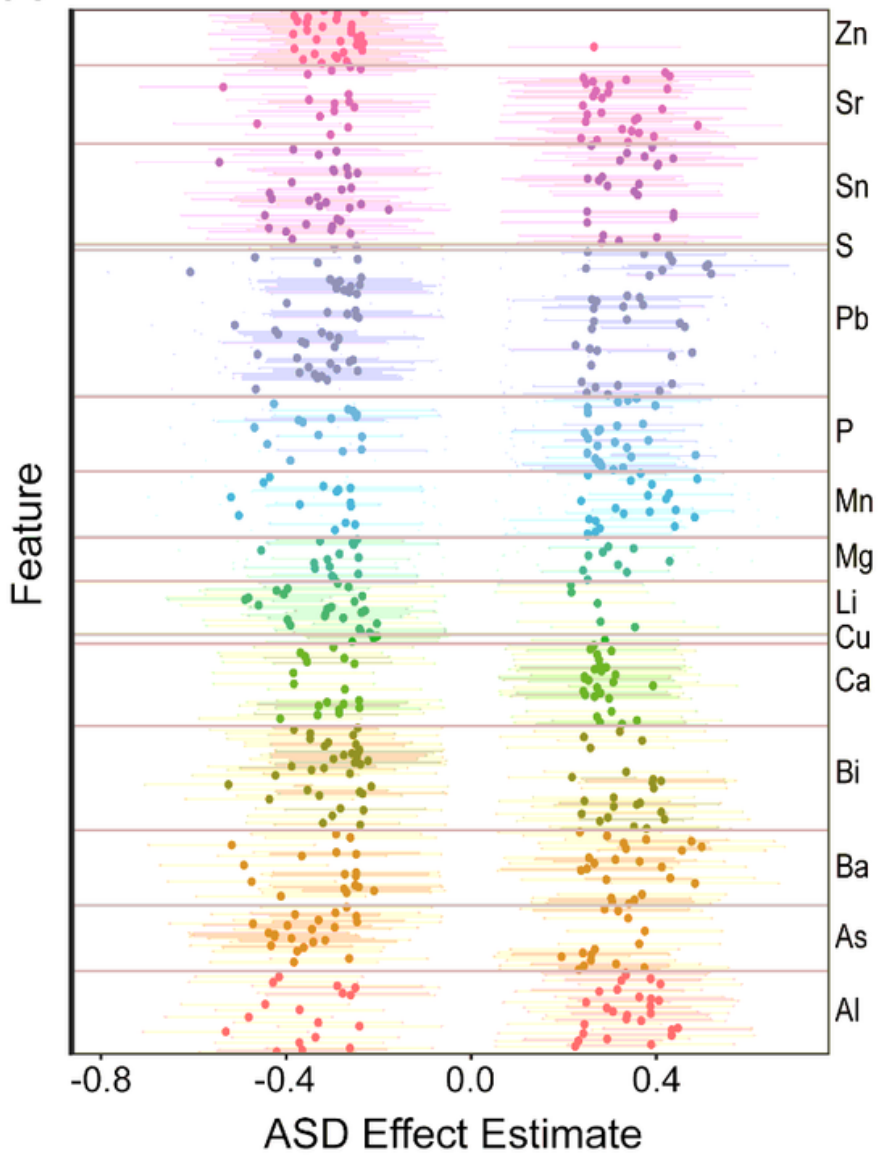

C

\begin{tabular}{|c|c|c|}
\hline Strata & $\begin{array}{l}\text { Performance } \\
(\mathrm{AUC}, 95 \% \mathrm{Cl})\end{array}$ & $\begin{array}{l}\text { Difference from } \\
\text { Overall }(P)\end{array}$ \\
\hline Overall & $0.92(0.86-0.97)$ & \\
\hline Male $\mathrm{Cr}$ & $0.92(0.85-0.98)$ & B) $P=0 . c$ \\
\hline Female Children & $0.91(0.79-1.00)$ & D) $P=0.88$ \\
\hline
\end{tabular}

B

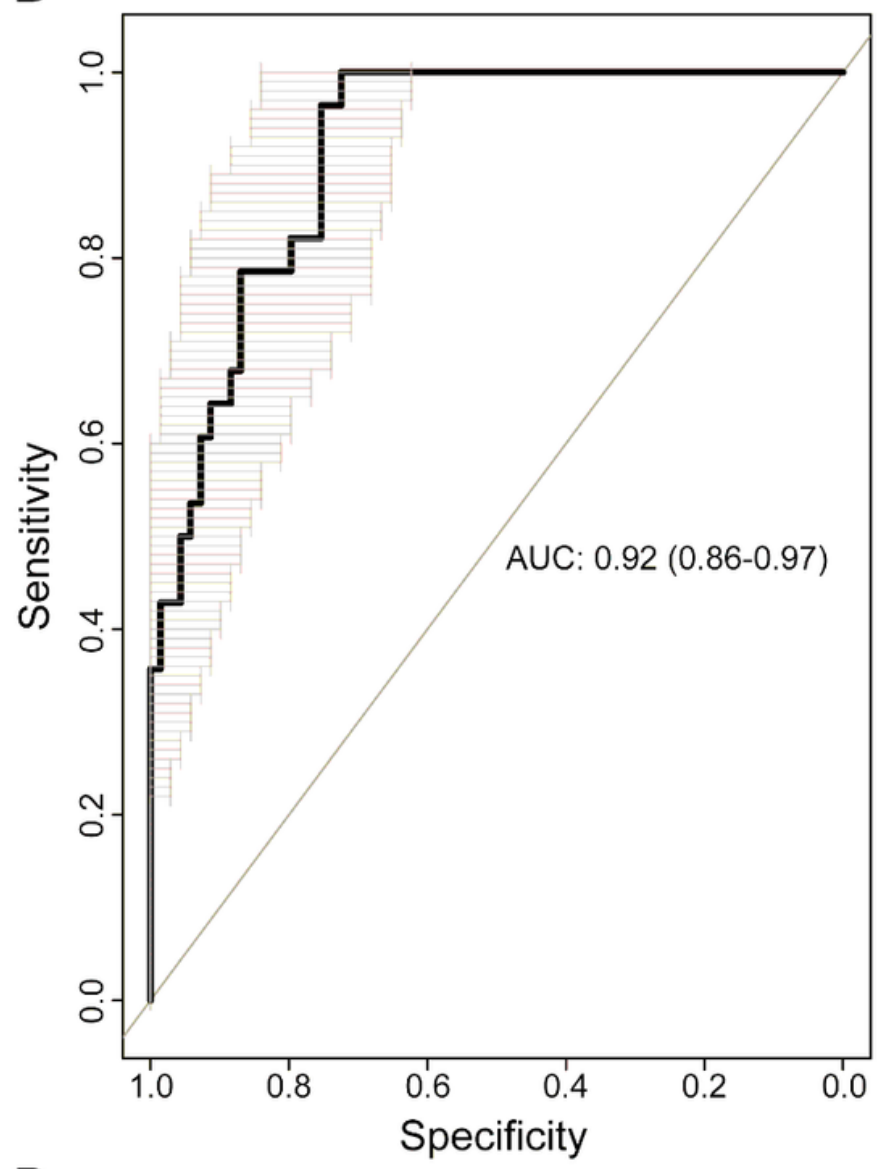

D

\begin{tabular}{lll} 
Strata & $\begin{array}{l}\text { Performance } \\
\text { (AUC, 95\% CI) }\end{array}$ & $\begin{array}{l}\text { Difference from } \\
\text { Overall }(P)\end{array}$ \\
\hline Overall & $0.92(0.86-0.97)$ & - \\
0-1.5 Years & $0.85(0.75-0.95)$ & $P=0.20$ \\
1.5-12 Years & $0.97(0.89-1.00)$ & $P=0.87$ \\
12-21 Years & $1.00(1.0-1.0)$ & $P=1.00$ \\
\hline
\end{tabular}

Figure 2

Elemental pathways associated with ASD diagnosis. (A) For each of 210 features measured in each elemental pathway, we constructed a discrete generalized linear model to test for associations with odds of diagnosis with ASD. Models were adjusted for age and sex, and p-values associated with diagnostic status were adjusted via false discovery rates (FDR). X-axis plots the standardized effect estimate for the effect of ASD diagnosis; dots and corresponding lines reflect the effect estimate and associated $95 \%$ confidence interval for each feature. (B) Receiver Operating Characteristic (ROC) curve generated from predicting case status in the validation set ( $\mathrm{N}=97 ; 28$ ASD). (C) Comparison of Overall Model Performance with Sex-Stratified Estimates of Model Performance. P-values reflect comparison of sexstratified ROC curves relative to ROC curves in the overall model. (D) Comparison of Overall Model Performance with Age-Stratified Estimates of Model Performance. P-values reflect comparison of agestratified ROC curves relative to ROC curves derived the overall model. 


\section{Supplementary Files}

This is a list of supplementary files associated with this preprint. Click to download.

- SupplementalMaterialHairASDmanuscriptJan24.docx 